\title{
The XMM-Newton view of stellar coronae: Coronal structure in the Castor $\mathrm{X}$-ray triplet ${ }^{\star}$
}

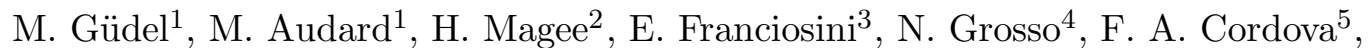 \\ R. Pallavicini ${ }^{3}$, and R. Mewe ${ }^{6}$ \\ 1 Paul Scherrer Institut, Würenlingen \& Villigen, 5232 Villigen PSI, Switzerland \\ 2 Mullard Space Science Laboratory, University College London, Holmbury St. Mary, Dorking, Surrey, RH5 6NT, UK \\ 3 Osservatorio Astronomico di Palermo "G. S. Vaiana", Piazza del Parlamento 1, 90134 Palermo, Italy \\ 4 Max-Planck-Institut für Extraterrestrische Physik, PO Box 1312, 85741 Garching, Germany \\ 5 University of California, Santa Barbara, CA 93106, USA \\ ${ }^{6}$ SRON Laboratory for Space Research, Sorbonnelaan 2, 3584 CA Utrecht, The Netherlands
}

Received 2 October 2000 / Accepted 20 October 2000

\begin{abstract}
We present results of a comprehensive X-ray investigation of the multiple stellar system Castor AB + YY Gem. The observations were obtained with the XMM-Newton observatory. We report the first spatially separated detection and identification of both Castor A and B as frequently flaring X-ray sources. Spectral modeling of the YY Gem and Castor coronal sources indicates a broad plasma distribution between 2-15 MK. Elemental abundances are found to be below solar photospheric values, with the abundance of Ne being highest $(\sim 0.7$ times solar photospheric). The abundances increase during a large flare, most notably for Fe. The O viI He-like line triplet indicates average coronal densities of the cooler plasma of a few times $10^{10} \mathrm{~cm}^{-3}$ for YY Gem and Castor. The YY Gem light curve shows three deep eclipses, indicating that the coronae of both binary components are similarly active and are relatively compact. A 3-D light curve inversion shows that coronal active regions follow density scale heights compatible with spectroscopically measured temperatures. The dominant densities also agree with spectroscopic values. Most active regions are located between \pm 50 degrees latitude, in agreement with findings from previous Doppler imaging. We also report the tentative detection of line broadening due to orbital motion.
\end{abstract}

Key words. stars: activity - stars: coronae - stars: flare - stars: abundances - stars: binaries: eclipsing - stars: individual: YY Gem, Castor

\section{Introduction}

Close binary systems are of great value for the study of atmospheric structuring. Although short-period binaries are often tidally locked, the mutual influence of the components on the internal structure and dynamo is poorly understood; it appears that the principal effect of binarity is the enforced rapid rotation of the components, which generates strong magnetic fields through a dynamo (Guinan \& Giménez 1993).

Comparison between coronal properties of binary components and single, active field stars potentially provides information on the generation of the dynamo. The ubiquitous magnetic activity in close binary stars suggests the

Send offprint requests to: M. Güdel, e-mail: guedel@astro.phys.ethz.ch

* Based on observations obtained with XMM-Newton, an ESA science mission with instruments and contributions directly funded by ESA Member States and the USA (NASA). presence of a strong magnetic dynamo in the stellar interiors which, according to current theory, is generated by convection and differential rotation. Given their strong tidal effects, however, close binary stars are expected to show little or no differential rotation. It thus seems that differential rotation plays a minor role in the generation of the dynamo, or that the stellar interiors rotate differentially despite the observed tidal locking of the photospheres (Guinan \& Giménez 1993). Eclipsing binaries further offer a unique advantage for the study of the spatial extent, the distribution, and the location of emitting material. Such systems may provide information on the location of the underlying dynamo.

Some stars or binaries indicate the presence of solarlike coronal structures that are comparable with solar active regions (Schmitt \& Kürster 1993), while observations of other systems suggest extended structures in particular between the components, perhaps related to intrabinary 
magnetic fields (Uchida \& Sakurai 1983; Siarkowski et al. 1996). Giant magnetic structures have been identified on RS CVn binaries through eclipse mapping (White et al. 1990). Spectral differences at different phases of the eclipse may indicate radial structuring, but reported results are ambiguous and contradictory (Walter et al. 1983; White et al. 1990; Ottmann et al. 1993).

Strong polar magnetic fields have been proposed for magnetically active stars (Schüssler \& Solanki 1992) based on arguments involving Coriolis forces in the stellar interior. Doppler imaging of active stars indeed suggests active regions concentrated at high latitudes (e.g., Strassmeier \& Rice 1998). Corresponding coronal geometries have also been reported from radio observations (Benz et al. 1998; Mutel et al. 1998) and from X-ray observations of a flaring region that was occulted during an eclipse of Algol (Schmitt \& Favata 1999). Radio Very Long Baseline Interferometry (VLBI) observations provide clues for very extended magnetospheres, including structures of the size of the intrabinary distance in binary systems (e.g., Mutel et al. 1985, 1998; Alef et al. 1997; Benz et al. 1998). The question then is whether such features are generally found on active stars, whether they contain hot plasma, whether they are preferred on single stars or on binaries, and whether there is additional strong activity at low latitudes. To address these questions, we have obtained a long observation of the Castor triplet of stellar binaries with the $X M M$-Newton observatory.

\section{The Castor system: Previous knowledge}

Castor is a hierarchical sextuplet system at a distance of $15.81 \pm 0.31 \mathrm{pc}(\mathrm{ESA} 1997)$. The principal optical components are Castor $\mathrm{A}(\mathrm{A} 1 \mathrm{~V})$ and Castor B (A5Vm) in an orbit with a period of $\sim 467$ yrs and a separation of presently 3. . 9 . Each component is accompanied by an invisible late-type star in a close orbit. Castor A's companion is most likely to be a late K star (Güdel \& Schmitt 1996) in a $9.21 \mathrm{~d}$ eccentric orbit, while Castor B's companion appears to be an early M dwarf (Güdel \& Schmitt 1996) in a $2.93 \mathrm{~d}$ circular orbit (orbit information from Batten et al. 1978; Heintz 1988; Haisch et al. 1990).

YY Gem (= Castor C) is a short-period spectroscopic binary and flare star (e.g., Haisch et al. 1990) located $71^{\prime \prime}$ south of the Castor system. It is gravitationally bound to Castor but only marginally so (Anosova \& Orlov 1991). It consists of two almost identical dMe stars in a circular $0.814 \mathrm{~d}$ orbit. Due to the fortunate orbit inclination angle of $86^{\circ} .2$ (Pettersen 1976), the system produces nearly total primary and secondary eclipses, where the primary has a slightly larger radius $\left(0.66\right.$ vs. $\left.0.58 R_{\odot}\right)$ and mass $(0.62$ vs. $0.57 M_{\odot}$ ) than the secondary (Haisch et al. 1990). Doppler imaging shows magnetic photospheric spot concentrations in mid-latitude bands (around $\pm 45^{\circ}$ ), with a weaker spot at the equator (Hatzes 1995).

YY Gem has been extensively observed in X-rays. Pallavicini et al. (1990) report quiescent and flaring emission not only from YY Gem itself, but they detect the
Table 1. Observing log on 2000 April 24/25

\begin{tabular}{llc}
\hline Instrument & UT range & JD range $2451659.0+$ \\
\hline RGS & $18: 20: 42-00: 49: 19$ & $0.26438-0.53425$ \\
& $02: 54: 14-21: 15: 19$ & $0.62100-1.38564$ \\
\multirow{2}{*}{ MOS } & $19: 22: 12-00: 45: 25$ & $0.30708-0.53154$ \\
& $03: 55: 40-20: 52: 16$ & $0.66366-1.36963$ \\
pn & $18: 42: 50-00: 47: 50$ & $0.27975-0.53322$ \\
& $03: 16: 26-20: 54: 41$ & $0.63641-1.37131$ \\
\hline
\end{tabular}

(unresolved) Castor AB system as a somewhat weaker $\mathrm{X}$-ray source as well. This led to some speculation about possible X-ray emission from A stars. Later observations obtained by ROSAT (PSPC \& HRI) and by ASCA addressed this problem further (Schmitt et al. 1994; Gotthelf et al. 1994). Castor AB was identified again, revealing a surprisingly high rate of flares. Positional analysis indicated that the emission is most probably coming from Castor A. The observed X-ray luminosity requires a mass $>0.4 M_{\odot}$ if a single star is responsible (Schmitt et al. 1994).

Schmitt et al. (1994) present the first high-resolution radio observations of the Castor system, revealing radio emission not only from YY Gem as reported earlier (Linsky \& Gary 1983), but also from Castor A, where the flux ratio $f_{\text {Castor }} / f_{\text {YYGem }}$ is similar to the $\mathrm{X}$-ray flux ratio. The discovery of the third radio source, coincident with Castor B, showed that all three binary systems are "magnetically active" (Güdel \& Schmitt 1996). VLBI studies revealed a coronal size of $210^{11} \mathrm{~cm}$ for YY Gem, corresponding to the intrabinary distance (Alef et al. 1997). The absence of a detected radio eclipse supports the large size. However, indications of deep eclipses were reported from X-ray observations (Haisch et al. 1990), clearly suggesting different structures for radio and X-ray emitting plasma.

\section{Observations and data analysis}

The observations reported here were obtained by $X M M$ Newton (Jansen et al. 2001) in two closely separated intervals (Table 1). Two EPIC MOS cameras (Turner et al. 2001) provide an angular resolution of $\sim 4^{\prime \prime}$, just comparable with the separation between Castor A and B. Both MOS were operated in the "partial window mode 2", thus including both Castor and YY Gem. The EPIC pn camera (Strüder et al. 2001) was operated in the small window mode, with Castor AB outside the field of view. The two RGS detectors (den Herder et al. 2001) were in spectroscopy mode. The optical monitor (Mason et al. 2001) was in a closed position, due to the strong optical light of Castor AB.

All data were reduced with the XMM-Newton Science Analysis System (SAS) software version 4.1, using several updates of individual tasks. For the RGS data, standard processing was performed using the RGSPROC task, followed by spectral extraction and response generation. The RGS fully separates Castor from 
L346
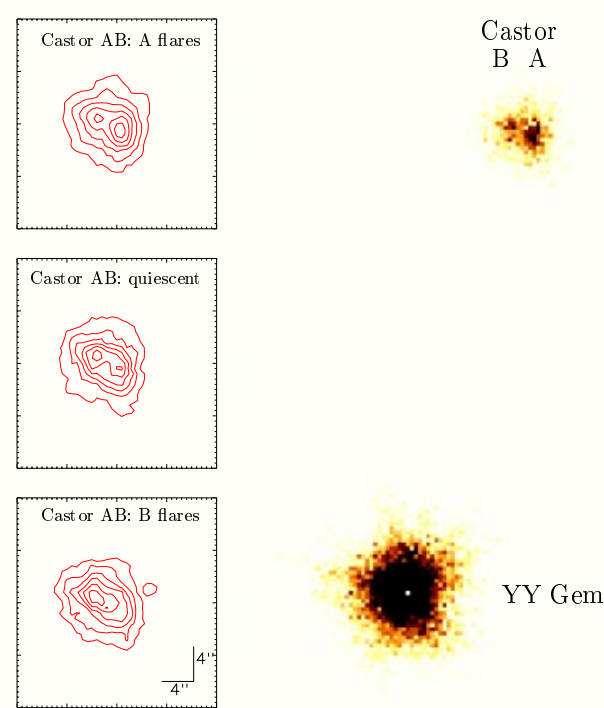

Fig. 1. EPIC MOS1 image of the Castor system. The components are labeled. Insets: contour plots of Castor AB data subsets. Contours are normalized relative to peak. Top: only flares on Castor A. Middle: low-level emission. Bottom: only flares on Castor B (see Fig. 2b)

YY Gem. The RGS spectral products were analysed in the Utrecht spectral software SPEX using the Collisional Ionization Equilibrium model CIE (Kaastra et al. 1996).

The EPIC MOS data were processed using EMPROC. For the spectral analysis, we used a response matrix provided by Turner et al. (2001). The EPIC pn data were reduced with EPPROC. A preliminary calibration was applied to the small window data. We used EVSELECT for source extraction. Spectral analysis was performed in XSPEC (Arnaud 1996) using multi- $T$ models for optically thin plasma with variable abundances (VMEKAL) that are compatible with the CIE model.

\section{Results}

\subsection{X-ray images and light curves of YY Gem and Castor $A B$}

The EPIC MOS show for the first time spatially separated images of Castor A and B and thus prove that both Castor binaries (i.e., most probably their low-mass companions) are X-ray sources (Fig. 1), compatible with their radio detections (Güdel \& Schmitt 1996). Their separation and position angle coincide with the optically determined values (Heintz 1988). Overall, Castor A is X-ray brighter. Both stars flare frequently (Fig. 2b), in fact at a surprisingly high rate, so that no part of the light curve is constant, with Castor B's flare rate being somewhat higher. A closer look at Fig. 2b shows that flares on Castor B reveal rapid decays, while on Castor A they decay less rapidly. The light curve of YY Gem will be discussed further below.
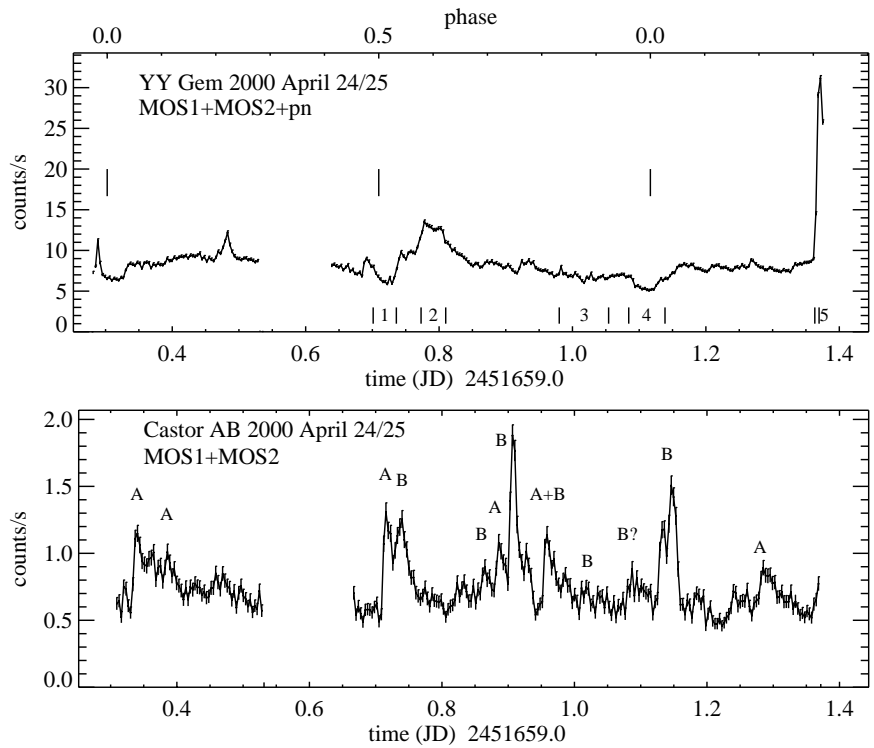

Fig. 2. Top: Co-added light curve of YY Gem from MOS1\&2 and pn binned to $300 \mathrm{~s}$ (the first $\sim 40 \mathrm{~min}$ of each segment were observed only by the pn camera; its count rate was adjusted by a factor of $\sim 1.7$ determined from later time intervals). The upper vertical bars indicate the eclipse times (primary-secondaryprimary), the lower bars give the intervals used for MOS fits (Sect. 4.3 and Fig. 7). Bottom: Light curve of Castor AB, derived from MOS1\&2. Labels (A or B) identify flaring star. Note the different vertical scales in the two figures

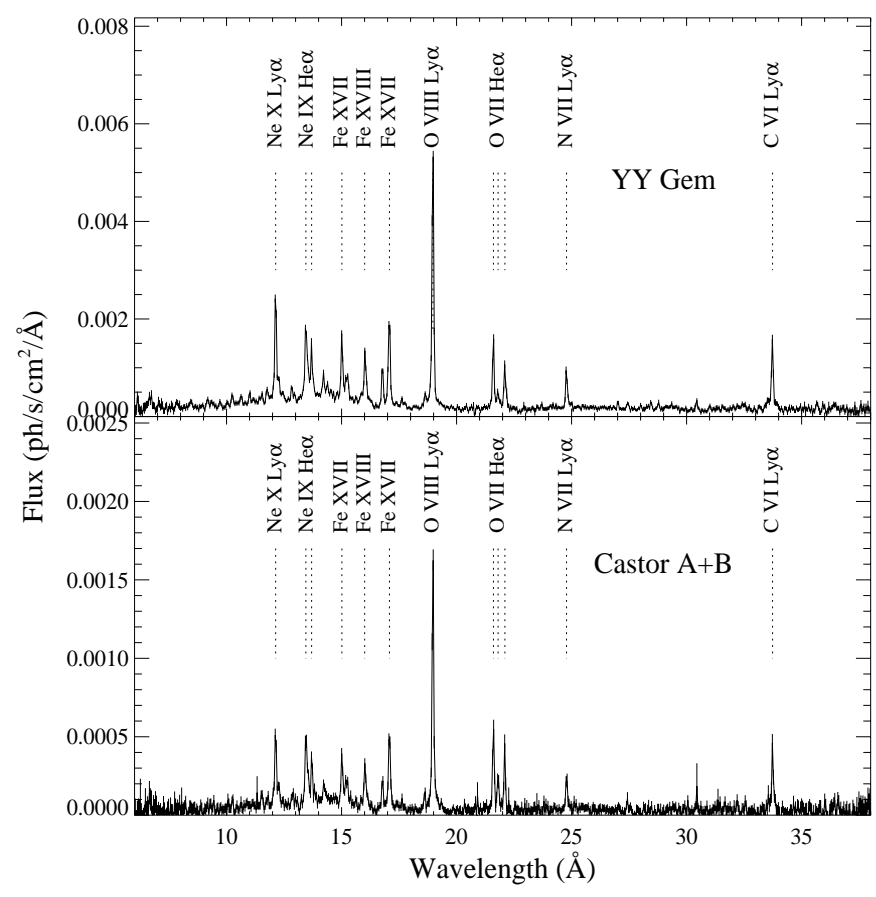

Fig. 3. RGS1\&2 fluxed spectra of $\mathrm{YY}$ Gem and Castor $\mathrm{AB}$ (bin resolution: $0.04375 \AA$ ). Note the different vertical scales in the two panels

\subsection{The RGS spectra, and density analysis}

Figure 3 shows the coadded and fluxed spectra of YY Gem and Castor AB from all RGS observations. 


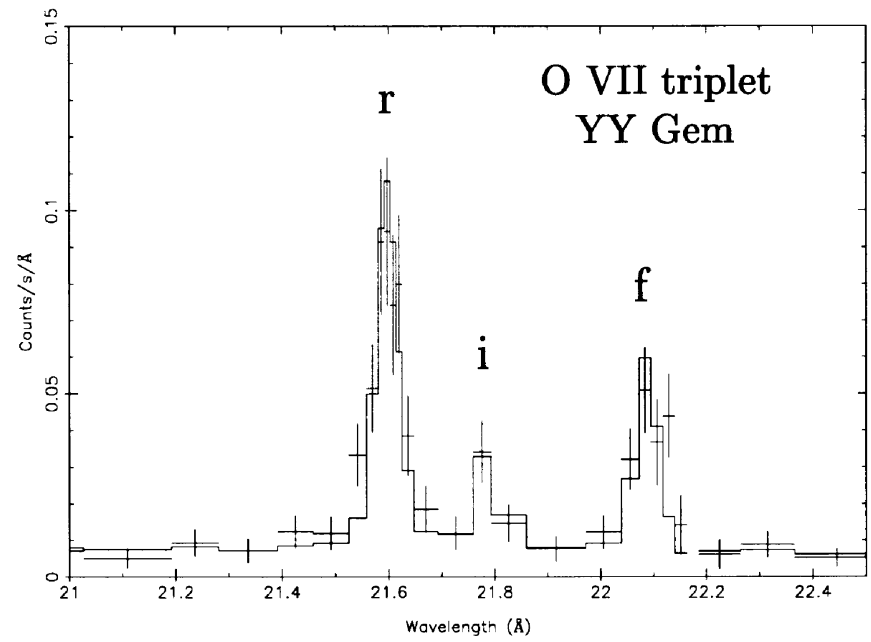

Fig. 4. Density-sensitive He-like O VII triplet of quiescent YY Gem

A series of lines of highly ionized $\mathrm{Fe}$ and several lines of the Ly and He series are visible, most notably from $\mathrm{C}$, $\mathrm{N}, \mathrm{O}$, and Ne. The significant continuum indicates the presence of hot plasma in both sources. The flux ratio between the forbidden $(f)$ and the intercombination $(i)$ lines of the He-like O VII triplet at $22 \AA$ is density sensitive in the range $10^{9}-10^{13} \mathrm{~cm}^{-3}$ (Mewe et al. 1985). We have fitted (Fig. 4) the triplet for the quiescent RGS1 spectrum of YY Gem in SPEX and found $n_{\mathrm{e}}=\left(2.9_{-2.5}^{+3.4}\right) 10^{10} \mathrm{~cm}^{-3}$, where the errors signify $90 \%$ confidence limits. To confirm this value, we derived the individual $f$ and $i$ fluxes by fitting the line spread function to the lines, to obtain a flux ratio $f / i=2.75 \pm 0.92$. Given that the emission is most probably originating from plasma with $T \approx 3 \mathrm{MK}$ (Sect. 4.3), the new calculations by Mewe et al. (2001) for the O VII triplet indicate $n_{\mathrm{e}}=\left(1.6_{-1.4}^{+2.6}\right) 10^{10} \mathrm{~cm}^{-3}$, in agreement with SPEX. For Castor, we find a similar value from SPEX, $n_{\mathrm{e}}=\left(1.6_{-1.6}^{+2.3}\right) 10^{10} \mathrm{~cm}^{-3}$, although it is not constrained toward low densities.

\subsection{Thermal structure and abundances}

We fitted separately RGS2, MOS and pn data for the quiescent YY Gem before the large flare at $1.35 \mathrm{~d}$ with $3-T$ coronal models (Table 2). For the MOS, the time interval was $t=1.157-1.351 \mathrm{~d}$ (Fig. 2a). For the RGS we included data between $t=0.85-1.19 \mathrm{~d}$ after which time a new RGS observing segment started. Both intervals show very similar hardness characteristics. We modeled the flare at $t>1.35 \mathrm{~d}$ for MOS and pn by keeping the quiescent model found before fixed, and adding one or two further thermal models, for which we fitted abundances, $T$, and $E M$ s. Multi- $T$ fit results are reported in Table 2.

All three fits to the quiescent spectra agree quite well. The most notable trends are low abundances for elements with a low First Ionization Potential (FIP), most clearly for Fe, while high-FIP elements, in particular Ne, show higher abundances. The poor result for $\mathrm{S}$ from the
Table 2. Model fits for RGS2, MOS1, and $\mathrm{pn}^{a}$

\begin{tabular}{|c|c|c|c|c|}
\hline \multirow[b]{2}{*}{ Parameter } & \multicolumn{3}{|c|}{$-Y Y$ Gem $Q U I E S C E N T-$} & \multirow{2}{*}{$\begin{array}{l}\text { Castor } A B \\
\text { MOS1 }\end{array}$} \\
\hline & RGS2 & MOS1 & pn & \\
\hline$\overline{k T_{1}}$ & $0.29_{-0.04}^{+0.05}$ & $0.37_{-0.05}^{+0.03}$ & $0.25_{-0.02}^{+0.02}$ & $0.17_{-0.07}^{+0.8}$ \\
\hline$k T_{2}$ & $0.66_{-0.03}^{+0.04}$ & $0.74_{-0.06}^{+0.05}$ & $0.60_{-0.02}^{+0.02}$ & $0.67_{-0.02}^{+0.02}$ \\
\hline$k T_{3}$ & $1.33_{-0.43}^{+\cdots}$ & $1.82_{-0.30}^{+0.42}$ & $1.12_{-0.10}^{+0.21}$ & $2.05_{-0.58}^{+\cdots}$ \\
\hline $\log E M_{1}{ }^{b}$ & $51.98_{-0.18}^{+0.12}$ & $52.07_{-0.16}^{+0.06}$ & $51.80_{-0.08}^{+0.10}$ & $50.79_{-}^{+0.4}$ \\
\hline $\log E M_{2}{ }^{b}$ & $52.26_{-0.13}^{+0.11}$ & $52.21_{-0.08}^{+0.07}$ & $51.98_{-0.09}^{+0.08}$ & $51.75_{-0.14}^{+0.13}$ \\
\hline $\log E M_{3}{ }^{b}$ & $51.67_{-0.70}^{+0.13}$ & $51.65_{-0.13}^{+0.08}$ & $51.85_{-0.11}^{+0.09}$ & $51.02_{-0.41}^{+0.19}$ \\
\hline $\mathrm{C}$ & $0.48_{-0.16}^{+0.30}$ & $=1$ & $=1$ & $=1$ \\
\hline $\mathrm{N}$ & $0.60_{-0.19}^{+0.35}$ & $=1$ & $=1$ & $=1$ \\
\hline $\mathrm{O}$ & $0.29_{-0.06}^{+0.13}$ & $0.35_{-0.04}^{+0.04}$ & $0.38_{-0.04}^{+0.04}$ & $0.22_{-0.11}^{+0.17}$ \\
\hline $\mathrm{Ne}$ & $0.75_{-0.15}^{+0.38}$ & $0.63_{-0.10}^{+0.09}$ & $0.74_{-0.29}^{+0.34}$ & $0.46_{-0.14}^{+0.19}$ \\
\hline $\mathrm{Mg}$ & $0.17_{-0.17}^{+0.27}$ & $0.17_{-0.07}^{+0.07}$ & $0.30_{-0.09}^{+0.10}$ & $0.26_{-0.10}^{+0.15}$ \\
\hline $\mathrm{Si}$ & $0.44_{-0.44}^{+0.73}$ & $\begin{array}{l}0.29_{-0.08}^{+0.09} \\
\end{array}$ & $0.42_{-0.08}^{+0.09}$ & $0.25_{-0.09}^{+0.11}$ \\
\hline S & $0.14_{-0.14}^{+0.23}$ & $0.27_{-0.16}^{+0.17}$ & $0.36_{-0.15}^{+0.08}$ & $0.47_{-0.28}^{+0.32}$ \\
\hline $\mathrm{Ca}$ & $=1$ & $=1$ & $=1^{-0.10}$ & $=1^{-0.20}$ \\
\hline $\mathrm{Fe}$ & $0.14_{-0.03}^{+0.05}$ & $0.15_{-0.02}^{+0.03}$ & $0.24_{-0.04}^{+0.05}$ & $0.12_{-0.04}^{+0.06}$ \\
\hline $\mathrm{Ni}$ & $0.21_{-0.21}^{+0.23}$ & $0.00_{-0.00}^{+0.16}$ & $=0.21$ & $0.13_{-0.13}^{+0.35}$ \\
\hline \multirow[t]{2}{*}{$\chi^{2} / \operatorname{dof}$} & $183 / 321$ & $186 / 140$ & $372 / 360$ & $160 / 130$ \\
\hline & $-Y Y G$ & mFLARE & $>1.35 d)$ & \\
\hline Parameter & MOS1 & MOS2 & pn & \\
\hline$k T_{1}$ & - & - & $0.77_{-0.06}^{+0.07}$ & \\
\hline$k T_{2}$ & $3.22_{-0.32}^{+0.32}$ & $3.47_{-0.35}^{+0.39}$ & $3.21_{-0.22}^{+0.27}$ & \\
\hline $\log E M_{1}{ }^{b}$ & - & $-\quad-0.30$ & $51.59_{-0.08}^{+0.11}$ & \\
\hline $\log E M_{2}{ }^{b}$ & $52.58_{-0.04}^{+0.03}$ & $52.54_{-0.05}^{+0.04}$ & $52.49_{-0.03}^{+0.03}$ & \\
\hline $\mathrm{O}$ & $0.15_{-0.15}^{+1.04}$ & $1.80_{-1.24}^{+1.54}$ & $1.53_{-0.52}^{+0.61}$ & \\
\hline $\mathrm{Ca}$ & $\begin{array}{l}3.50_{-3.26}^{+3.25} \\
+30\end{array}$ & $0.00_{-0.00}^{+2.24}$ & $4.83_{-2.71}^{+2.69}$ & \\
\hline $\mathrm{Fe}$ & $0.74_{-0.22}^{+0.25}$ & $\begin{array}{l}0.85_{-0.29}^{+0.36} \\
\end{array}$ & $0.74_{-0.15}^{+0.16}$ & \\
\hline$\chi^{2} /$ dof & $84 / 124$ & $123 / 123$ & $369 / 354$ & \\
\hline
\end{tabular}

a Errors give $90 \%$ confidence intervals based on $\chi_{\min }^{2}+2.706$. Some parameters were held fixed $("=x ")$. "..." indicate unconstrained parameters. Abundances are relative to solar photospheric abundances (Anders \& Grevesse 1989).

${ }^{b}$ Due to the present lack of Good Time Intervals for pn data, we normalized the count rates with the length of the time interval; this implies a lower limit for the $E M$ values for pn.

${ }^{c}$ These flare components are additional to the fixed quiescent 3- $T$ model. $E M$ s are averages over rise and flare peak.

RGS is due to weak lines with poor atomic data (Audard et al. 2001). Figure 5 shows an $E M$ distribution recovered from the RGS2 spectrum, using Chebychev polynomials of degree 5. A broad distribution from 2-15 MK compatible with the $3-T$ fit results is evident. There is little plasma with $T<2 \mathrm{MK}$ : a number of lines of $\mathrm{C}$ VI, N VI, N VII, and $\mathrm{O}$ VII with formation temperatures in this range constrain this regime. During the flare, some elemental abundances increase, in particular Fe which increases by a factor of 3-5. Note, however, that the significance of the flare abundance values for the other elements are clearly at the limit of the present calibration and the $\mathrm{S} / \mathrm{N}$ available.

Figure 6 illustrates spectral fits to the YY Gem pn data for quiescence and for the large flare. More detailed timeresolved spectroscopy was performed with MOS, with five segments selected as follows (Fig. 2a): (1) secondary eclipse - (2) flare peak at $\sim 0.8 \mathrm{~d}-(3)$ quiescent emission (around $1 \mathrm{~d}$ ) - (4) primary eclipse - (5) flare (including rise) at $1.37 \mathrm{~d}$. Two $T$ components were sufficient for the given $\mathrm{S} / \mathrm{N}$ ratio. The results are shown graphically in Fig. 7. The heating effects during the flares are evident. Note also the trend for higher metal abundances (in 


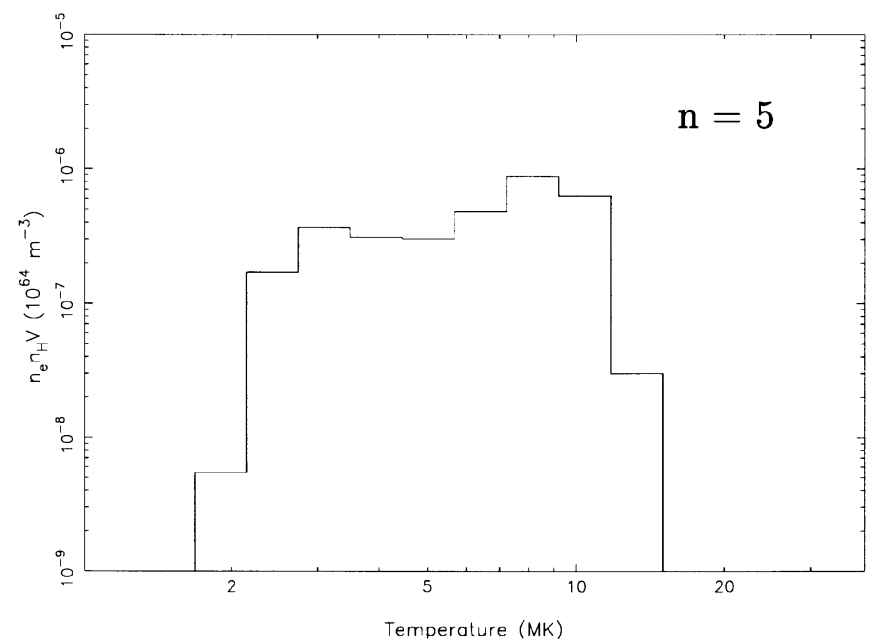

Fig. 5. $E M$ distribution for the quiescent YY Gem, derived from RGS2. A polynomial degree of 5 was used

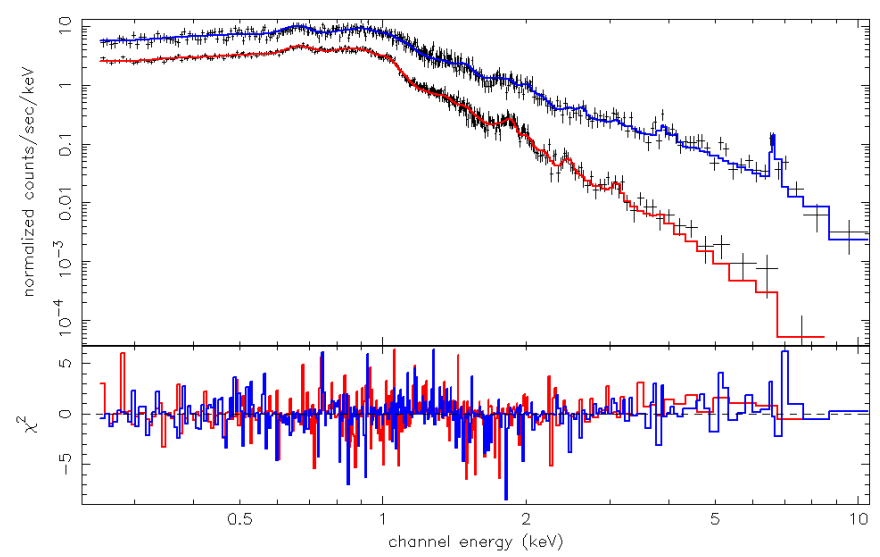

Fig. 6. YY Gem EPIC pn spectra (top: flaring; bottom: quiescent)

particular of $\mathrm{Fe}$ and $\mathrm{Ne}$ ) during the large flare, while no significant changes are seen during the eclipses.

How different is Castor AB from YY Gem? Close inspection of the RGS spectra in Fig. 3 reveal higher flux ratios of O VIII/O VII and Nex/NeIX for YY Gem than for Castor, indicating that YY Gem's corona is on average hotter despite the ongoing flaring on Castor. Some support for this result comes from multi- $T$ fits reported in Table 2 (from the integrated MOS1 observation). The ratio between the $E M \mathrm{~s}$ of the hottest to the intermediate component is larger for YY Gem. The abundance pattern is, within the errors, rather similar.

\subsection{Orbital motion of $Y Y$ Gem}

Radial velocity variations due to the orbital motion of the two stars in YY Gem may be evident as broadening of the spectral lines during the orbital period. Eclipse times and orbital phases were taken from Haisch et al. (1990) who predict a primary eclipse at JD $=2451660.11676$,
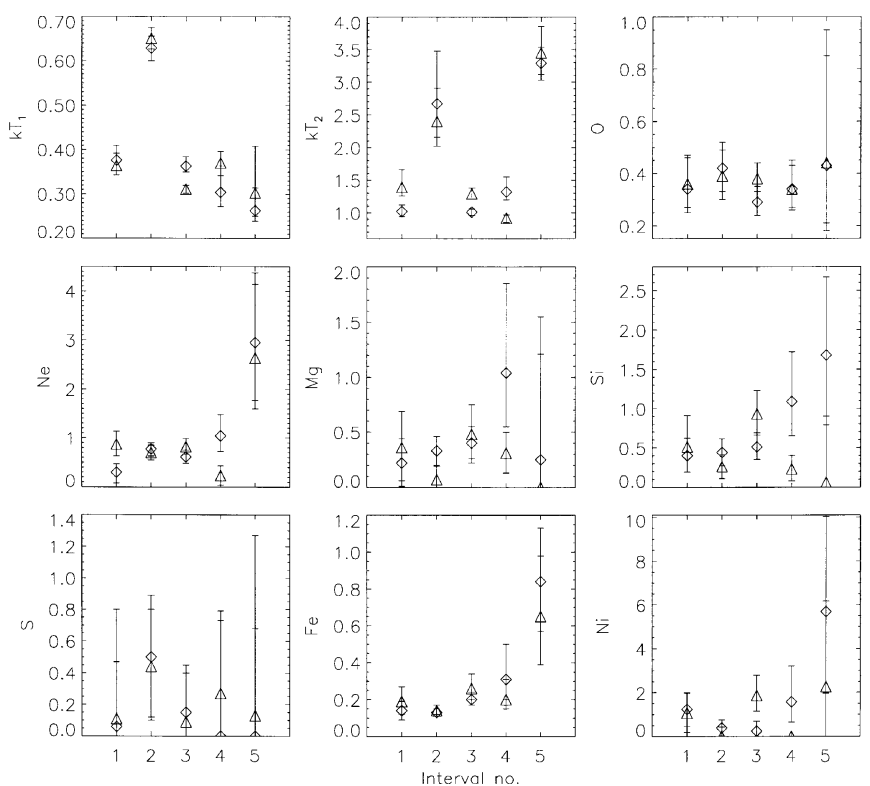

Fig. 7. Time resolved MOS1 (diamonds) and MOS2 (triangles) spectroscopy of YY Gem based on 2-T spectral fits, showing the parameters $k T_{1}, k T_{2}$, abundances of $\mathrm{O}, \mathrm{Ne}, \mathrm{Mg}, \mathrm{Si}, \mathrm{S}, \mathrm{Fe}$, and $\mathrm{Ni}$. The numbers on the $x$-axis identify time intervals (see text and Fig. 2a). Error bars indicate 90\% confidence ranges based on $\chi_{\min }^{2}+2.706$

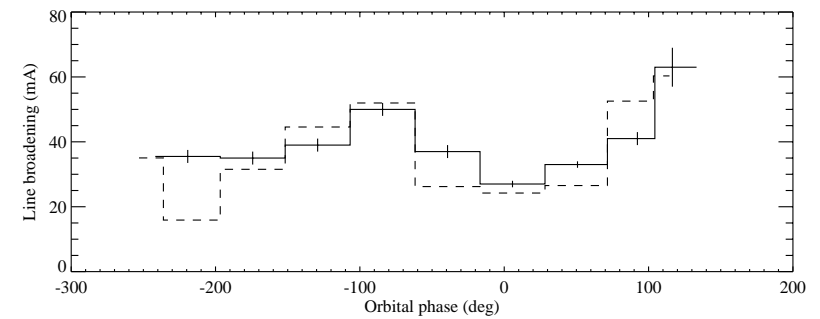

Fig. 8. Line broadening of the O viII Ly $\alpha$ line as a function of orbital phase, for RGS2. Two methods were applied: fit with the line spread function (dashed) and with a double-Gaussian profile (solid)

consistent with the three eclipses seen in the $X M M$ Newton observations (Fig. 2a).

The RGS spectra for the second observation were subdivided into time segments for which maximum or minimum line broadening would be expected (i.e. around quadrature $-90^{\circ}$ or $90^{\circ}$ and conjunction $0^{\circ}$ and $-180^{\circ}$ ). Two segmentations were used, firstly the observation was divided into 8 segments with segment 6 centered on the primary eclipse. Each segment, with the exception of segment 1, was approximately $8800 \mathrm{~s}$ in duration. A second set of time segments, shifted by about $2200 \mathrm{~s}$ relative to the first, was also constructed which optimized the counts in the first segment. Line broadening and centroids for the strongest emission line in the spectrum, that of $\mathrm{O}$ VIII at $18.969 \AA$, were determined firstly with SPEX using the calibrated line spread function in the response matrix for the RGS and secondly within the software package DIPSO (Howarth et al. 1998) by correcting for the effective area 
variation and fitting a combination of two Gaussian functions, which was found to give an acceptable representation of the line spread function.

The variation of line broadening for RGS2 (average for the two segmentations) for each of the methods used is shown in Fig. 8. It shows the expected sinusoidal variation with orbital phase, with minimum broadening occurring near conjunction (phases of $0^{\circ}$ and $-180^{\circ}$ ). It should be noted that the value for the 8 th segment was determined without including the flare. The amplitude of the variation is approximately $12 \mathrm{~m} \AA$, corresponding to a velocity of $190 \mathrm{~km} \mathrm{~s}^{-1}$ which is in reasonable agreement with the expected value of up to $160 \mathrm{~km} \mathrm{~s}^{-1}$, determined from the orbital motion (including the stellar sizes). No anomalous broadening, resulting from the inhomogeneity seen in the light curve modeling (Sect. 5), was detected.

The sinusoidal variation is not clearly evident in the results obtained from the RGS1 spectra. Although there is some variation during the observation it is not clearly correlated with the orbital period. We note, however, that although the detailed description of the response of both spectrometers is expected to evolve over time, we have preferred RGS2 for the present analysis as this description is more advanced than for RGS1.

\subsection{An eclipsed flare?}

A closer look at Fig. 2a may suggest that the small flare at $0.7 \mathrm{~d}$ is merely the beginning of a larger flare that is subsequently eclipsed and that "reappears" at $0.78 \mathrm{~d}$. Note that after the eclipse, a plateau (at $0.75 \mathrm{~d}$ ) first appears, which we interpret as being the end of the eclipse of the bulk "quiescent" material, while the flare, then required to be located about 80 degrees away in longitude from the substellar point, reappears later. Proving this scenario is difficult. We have nevertheless tentatively estimated location and extent of such a structure, both from the "ingress" and "egress" durations and the duration of the occultation. The optimum latitude is approximately -10 degrees. An appreciable height is required to explain the long occultation (up to $\sim 0.8 R_{*}$ above the photosphere). The longitudinal extent is of the same order (i.e., $[2-3] 10^{10} \mathrm{~cm}$ ).

\section{Eclipse modeling}

The long light curve available for YY Gem includes three eclipses (two primary and one secondary eclipse). We have applied a method first presented by Siarkowski (1992) and Siarkowski et al. (1996) for a 3-D deconvolution of X-ray light curves. In short, the stellar environment is divided into regular volume bins that are occupied by variable amounts of $E M$. After each iteration step, the model and the observed light curves are compared. For each phase, the EMs of all visible volume elements are increased by a factor equal to the ratio of observed flux to modeled flux. We have slightly modified the original procedure by applying volume bins defined in spherical coordinates. Bins are located at positions $\left(r_{i}, \theta_{i}, \phi_{i}\right)$. In the radial direction, we subdivide the interval from $R_{*}$ to $1.99 R_{*}$ for the primary (2.15 $R_{*}$ for the secondary) into 16 regular bins. In $\theta$ (co-latitude) direction, we define 36 equidistant bins from 0 (north pole) to $\pi$ (south pole). Finally, the width of the volume bins in $\phi$ (longitude) direction is such that all volumes at a given radius are equal, i.e., $\Delta \phi \propto \sin \theta$, with 72 equidistant bins at the equator. To prevent ambiguities at the pole, we keep at least four $\phi$ bins at each co-latitude.

Several words of caution are in order. i) The light curve clearly shows flares which must not enter into the modeling process. We therefore removed obvious flares at $0.48 \mathrm{~d}$, $0.7 \mathrm{~d}, 0.8 \mathrm{~d}$, and $1.37 \mathrm{~d}$ (Fig. 2a), and linearly interpolated from the pre-flare to the post-flare count rate. The gap around $0.6 \mathrm{~d}$ was also linearly interpolated. This is a serious limitation of the present modeling also in the light of the possible presence of further small flares. We selected the interval $0.55-1.36 \mathrm{~d}$ for modeling, corresponding to one orbit period. ii) Light curve inversion is an illposed problem and generally satisfies multiple solutions (Siarkowski 1992; Siarkowski et al. 1996). Physically reasonable spatial restrictions should be introduced to confine the problem to sensible solutions. We have done so by restricting the radial extent of the coronae to $\sim 1 R_{*}$ above the photosphere but, as we will see, this constraint will automatically be fulfilled for most of the $E M$. iii) There is much correlation between neighboring bins, depending on the time resolution and the signal-to-noise ratio of the measurements. The finest structures in the solutions are ambiguous or not significant. iv) Since the observer is located close to the orbital plane, some north-south ambiguity could be introduced, i.e., features are mirrored at the equator. Some features may also be found on both stars at similar coordinates relative to the star centers.

With these caveats in mind, our goal is not to reconstruct a real coronal image but to investigate statistical properties of physically reasonable solutions that are compatible with the light curve. While the most stringent restrictions are due to the eclipses, the most difficult part of the modeling is the underlying quasi-steady emission. Any axisymmetric distribution with matching total luminosity can reproduce this emission if it is sufficiently extended or lies close to the uneclipsed north poles of the stars. We have studied three special but suggestive cases that are specified as starting conditions: i) a diffuse underlying corona with $n_{\mathrm{e}} \propto r^{-1}$; ii) a diffuse underlying corona with $n_{\mathrm{e}}=$ const. iii) Addition of a strong, uneclipsed active region at each of the two north poles.

Case (iii) suppresses diffuse axisymmetric components during the iteration, essentially leaving the inhomogeneous structure for modeling. The latter was found identically from all three starting conditions. Figure 9 illustrates a possible model for one orbital revolution of the two stars as seen from the Earth (solution for case [i]; the primary star is held in the center). Note that

- the coronal plasma is inhomogeneous. This accounts for the asymmetric, deep, and short eclipses, and also for the eclipse profile; 

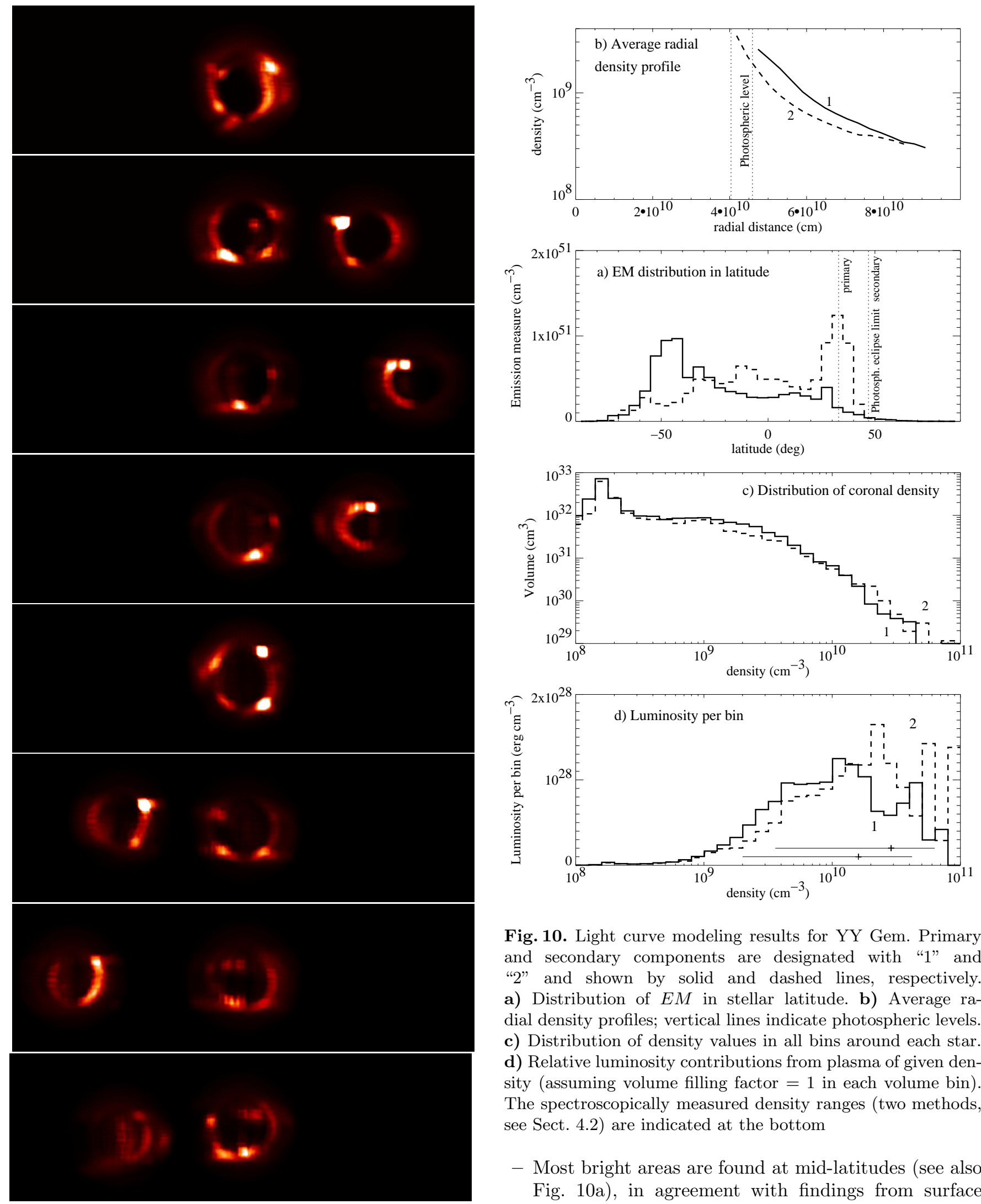

Fig. 10. Light curve modeling results for YY Gem. Primary and secondary components are designated with "1" and "2" and shown by solid and dashed lines, respectively. a) Distribution of $E M$ in stellar latitude. b) Average radial density profiles; vertical lines indicate photospheric levels. c) Distribution of density values in all bins around each star. d) Relative luminosity contributions from plasma of given density (assuming volume filling factor $=1$ in each volume bin). The spectroscopically measured density ranges (two methods, see Sect. 4.2) are indicated at the bottom

- Most bright areas are found at mid-latitudes (see also Fig. 10a), in agreement with findings from surface Doppler imaging (Hatzes 1995).

Fig. 9. Model for the coronal structure in the dMe binary YY Gem derived from light curve inversion. The primary star is in the center and is eclipsed by the secondary at phase 0 . The panels show the aspect in phase steps of $45^{\circ}$, starting with phase 0 (top; primary eclipsed)

After the modeling process, we removed the undetermined north polar active region and investigated the statistical and geometric properties of the solution (Fig. 10). The average density profile of the inhomogeneous part was 
computed (Fig. 10b) for each star. Evidently, most of the emitting material is closely concentrated near the stellar surfaces, and the density rapidly decreases outwards. The scale height is $\sim(1-4) 10^{10} \mathrm{~cm}$. The density scale height of an isothermal plasma is

$\Lambda=\frac{k T}{\mu m_{\mathrm{H}} g}=\frac{k T}{\mu m_{\mathrm{H}}} \frac{R_{*}^{2}}{G M_{*}}$

where $k$ is the Boltzmann constant, $m_{\mathrm{H}}$ is the mass of the hydrogen atom, $\mu \approx 0.67$ the mean molecular weight, and $G$ the gravitational constant. With the stellar parameters $R_{*}$ and $M_{*}$, we find a mean temperature of $\log T=6.5-7.1[\mathrm{~K}]$, in good agreement with the spectroscopic measurements (Fig. 5).

Figure 10c shows the statistical distribution of densities in all bins considered. While most bins are of low density (i.e., the high-density plasma has a small filling factor), the emissivity of a plasma scales with $n_{\mathrm{e}}^{2}$ so that most of the observed emission stems from plasma with densities of $\log n_{\mathrm{e}} \approx 9.7-10.5$ (Fig. 10d). This is again in gratifying agreement with the explicit density measurements in the RGS spectra. Finally, the total luminosities of the two stars are very similar: the non-axisymmetric portions modeled above contribute $1.310^{29} \mathrm{erg} \mathrm{s}^{-1}$ and $1.510^{29} \mathrm{erg} \mathrm{s}^{-1}$ from the primary and the secondary, respectively. The total system luminosity is $3.710^{29} \mathrm{erg} \mathrm{s}^{-1}$ (as determined from the MOS data).

\section{Summary and conclusions}

We have addressed a number of issues relevant for stellar coronal structure by making use of a high-quality $X M M$-Newton observation of the Castor/YY Gem system. The eclipse observations allow us to assess the statistical properties of the coronal plasma, such as its density scale height and the location of strongly emitting features. Our 3-D light curve inversion shows excellent agreement with spectroscopic results: i) the average density scale height implies a temperature that is in good agreement with the range measured from the available spectroscopy; ii) densities that dominate the X-ray emissivity agree well with spectroscopically determined average densities of a few times $10^{10} \mathrm{~cm}^{-3}$. iii) The active regions are mainly distributed between \pm 50 degrees in latitude, in agreement with previous findings from Doppler imaging (Hatzes 1995). The preference of mid-latitude regions is at variance with reports on polar magnetic spots on active stars. We may only speculate here whether the tidal interaction in this close system of almost fully convective M stars plays a role for this scenario. On the other hand, eclipse modeling does not exclude the presence of active regions near the north polar regions of the system.

At any rate, there is clear evidence that the coronae are highly structured and that both components are similarly active: i) Deep eclipses require similar amounts of $E M$ on both stars, and relatively compact active regions. We find no indications for very extended magnetospheres as inferred from radio observations (Alef et al. 1997). This is, in retrospect, not surprising: radio emission is due to high-energy electrons trapped in magnetic fields; they radiate more efficiently in a low-density environment where they suffer fewer collisions. On the other hand, efficient $\mathrm{X}$-ray emission originates preferentially in high-density environments since the emissivity scales with $n_{\mathrm{e}}^{2}$. Such places are naturally found closer to the stellar surfaces. ii) The asymmetries seen in the eclipse light curves clearly require structured coronae. And iii) we see indications for periodic line broadening, compatible in phase and amplitude with two orbiting stars of similar brightness.

We have investigated the thermal and elemental abundance structure both during quiescence and during flares. We find a broad distribution of $E M$ above $2 \mathrm{MK}$, with little material below. The $E M$ distribution peaks at about $8 \mathrm{MK}$, with a tail that may reach $15-20 \mathrm{MK}$ as derived from EPIC data. There is no appreciable change during the eclipses, but we do see significant increases of some abundances during the larger flare that reaches temperatures around $40 \mathrm{MK}$.

Finally, we report the definitive detection and identification of both Castor A and B as flaring X-ray sources. Although the Castor AB source was detected in X-rays before (Pallavicini et al. 1990; Schmitt et al. 1994; Gotthelf et al. 1994), the individual coronal components have hitherto been detected only as radio sources (Güdel \& Schmitt 1996). The new detections support the view that both Castor A and B have low-mass companions that are magnetically very active, as judged from the high flare rate.

Acknowledgements. We thank Slava Zavlin (MPE) who provided the CTI model for the PN small window mode. Frank Haberl (MPE) processed the pn data and made events files available to us. M. A. acknowledges support from the Swiss National Science Foundation, (grants 2100-049343 and 2000058827), from the Swiss Academy of Sciences, and from the Swiss Commission for Space Research. H. M. acknowledges financial support from PPARC. E. F. and R. P. acknowledge support from the Italian Space Agency. N. G. is supported by the European Union (Marie Curie Individual grant; HPMFCT-1999-00228). F. A. C. has been supported for data analysis by NASA grant NAS5-97119. SRON is supported financially by NWO.

\section{References}

Alef, W., Benz, A. O., \& Güdel M. 1997, A\&A, 317, 707

Anders, E., \& Grevesse, N. 1989, Geochim. Cosmochim. Acta, 53, 197

Anosova, J. P., \& Orlov, V. V. 1991, A\&A, 252, 123

Arnaud, K. A. 1996, in ASP Conf. Ser. 101, Astronomical

Data Analysis Software and Systems V, ed. G. Jacoby, \&

J. Barnes (San Francisco: ASP), 17

Audard, M., Behar, E., Güdel, M., et al. 2001, A\&A, 365, L329

Batten, A. H., Fletcher, J. M., \& Mann, P. J. 1978, Publ.

Dominion Astrophys. Obs., 15, 121

Benz, A. O., Conway, J., \& Güdel, M. 1998, A\&A, 331, 596

ESA, 1997, in European Space Agency SP-1200

Gotthelf, E. V., Jalota, L., Mukai, K., \& White, N. E. 1994, ApJ, 436, L91 
Güdel, M., \& Schmitt, J. H. M. M. 1996, in Radio Emission from the Stars and the Sun, ed. A. R. Taylor, \& J. M. Paredes (San Francsisco: ASP), 315

Guinan, E. F., \& Giménez, A. 1993, in The Realm of Interacting Binary Stars, ed. J. Sahade, et al. (Dordrecht: Kluwer), 51

Haisch, B. M., Schmitt, J. H. M. M., Rodonò, M., \& Gibson D. M. 1990, A\&A, 230, 419

Hatzes, A. P. 1995, in Stellar Surface Structure - Poster Proceedings, ed. K. G. Strassmeier, Vienna, 90

Heintz, W. D. 1988, PASP, 100, 834

den Herder, J. W., Brinkman, A. C., Kahn, S. M., et al. 2001, A\&A, 365, L7

Howarth, I. D., Murray, M. J., \& Mills, D. 1998, SERC Starlink User Note, 50

Jansen, F., Lumb, D., Altieri, B., et al. 2001, A\&A, 365, L1

Kaastra, J. S., Mewe, R., \& Nieuwenhuijzen, H. 1996, in UV and X-ray Spectroscopy of Astrophysical and Laboratory Plasmas, ed. K. Yamashita, \& T. Watanabe (Tokyo: Univ. Acad. Press), 411

Linsky, J. L., \& Gary, D. E. 1983, ApJ, 274, 776

Mason, K. O., Breeveld, A., Much, R., et al. 2001, A\&A, 365, L36

Mewe, R., Gronenschild, E. H. B. M., \& van den Oord, G. H. J. 1985, A\&AS, 62, 197

Mewe, R., Porquet, D., Raassen, A. J. J., \& Dubau, J. 2001, in preparation

Mutel, R. L., Lestrade, J. F., Preston, R. A., \& Phillips, R. B.
1985, ApJ, 289, 262

Mutel, R. L., Molnar, L. A., Waltman, E. B., \& Ghigo, F. D. 1998, ApJ, 507, 371

Ottmann, R., Schmitt, J. H. M. M., \& Kürster, M. 1993, ApJ, 413, 710

Pallavicini, R., Tagliaferri, G., Pollock, A. M. T., Schmitt, J. H. M. M., \& Rosso, C. 1990, A\&A, 227, 483

Pettersen, B. R. 1976, in Catalogue of Flare Star Data, Rept. No. 46 (Oslo: Inst. of Theoretical Astrophysics)

Schmitt, J. H. M. M., \& Favata, F. 1999, Nat, 401, 44

Schmitt, J. H. M. M., \& Kürster, M. 1993, Sci, 262, 215

Schmitt, J. H. M. M., Güdel, M., \& Predehl, P. 1994, A\&A, 287,843

Schüssler, M., \& Solanki, S. K. 1992, A\&A, 264, L13

Siarkowski, M. 1992, MNRAS, 259, 453

Siarkowski, M., Prés, P., Drake, S. A., White, N. E., \& Singh, K. P. 1996, ApJ, 473, 470

Strassmeier, K. G., \& Rice, J. B. 1998, A\&A, 339, 497

Strüder, L., Briel, U. G., Dennerl, K., et al. 2001, A\&A, 365, L18

Turner, M. J. L., Abbey, A., Arnaud, M., et al. 2001, A\&A, 365, L27

Uchida, Y., \& Sakurai, T. 1983, in Activity in Red Dwarf Stars, ed. M. Rodonó, \& P. Byrne (Dordrecht: Reidel), 629

Walter, F. M., Neff, J. E., Gibson, D. M. 1983, A\&A, 186, 241

White, N. E., Shafer, R. A., Parmar, A. N., Horne, K., \& Culhane, J. L. 1990, ApJ, 350, 776 